\title{
Metrological Software Test for Simulating the Method of Determining the Thermocouple Error in Situ During Operation
}

\author{
Jingliang Chen ${ }^{1}$, Jun $\mathrm{Su}^{1}$, Orest Kochan ${ }^{1,2}$, Mariana Levkiv ${ }^{3}$ \\ ${ }^{1}$ Country School of Computer Science, Hubei University of Technology, Wuhan, China \\ ${ }^{2}$ Department of Measuring Information Technologies, Lviv Polytechnic National University, Bandera Str. 28A, 79013, Lviv, \\ Ukraine \\ ${ }^{3}$ I. Horbachevsky Ternopil State Medical University, Maidan Voli 1, 46001, Ternopil, Ukraine, levkiv.mo@gmail.com
}

\begin{abstract}
The simplified metrological software test (MST) for modeling the method of determining the thermocouple (TC) error in situ during operation is considered in the paper. The interaction between the proposed MST and a temperature measuring system is also reflected in order to study the error of determining the TC error in situ during operation. The modelling studies of the random error influence of the temperature measuring system, as well as interference magnitude (both the common and normal mode noises) on the error of determining the TC error in situ during operation using the proposed MST, have been carried out. The noise and interference of the order of 5-6 $\mu \mathrm{V}$ cause the error of about $0.2-0.3^{\circ} \mathrm{C}$. It is shown that high noise immunity is essential for accurate temperature measurements using TCs.
\end{abstract}

Keywords: Thermocouple, determination of TC error in situ, determination of TC error during operation, metrological software test, simulation of measuring channel.

\section{INTRODUCTION}

Measurement plays an important role in science, industry, and commerce. The influence of metrology can be felt in technics [1], [2], technology [3], [4], economy [5], [6] and science [7]-[9], as well as in the fields of public health and safety [10], law and legal system and forensic studies [11], and in many others. Thus, it is no wonder that a considerable share of GDP in the developed countries belongs to the field of metrology and adjacent fields [12].

There are many measurement techniques and sensors [13] but the temperature is one of the most frequently measured physical quantities. Therefore, thermometry is a very important branch of metrology, and it also has numerous sensors [13]-[16] and techniques [17]-[19]. The importance of thermometry is reflected in the fact that the unit of temperature is one of the basic units of the International System of Units. Therefore, a lot of attention is dedicated to studying trends of thermometry [20] and improvement of the existing temperature scale [21].

Thermocouples (TCs) dominate in temperature measurements within the interval from $600^{\circ} \mathrm{C}$ to $1500^{\circ} \mathrm{C}$ [22]. However, they have a lot of sources of error: (i) electromagnetic interference [23]; (ii) data acquisition modules [24]; (iii) the reference junction [25]; (iv) nonlinearity of the conversion characteristic (CC) [22], [26]; (v) transient processes [22]; (vi) the initial deviation of CC from the nominal one (also called tolerance) at $800^{\circ} \mathrm{C}$ reaches $6^{\circ} \mathrm{C}$ [22], [26]; (vii) degradation of TC legs with operating time which leads to their longitudinal inhomogeneity [26]-[28], which is considered the biggest source of error. The latter source causes two interrelated errors: (i) the error due to drift of TC CC, which manifests itself in a stable condition of operation as a gradual change of a CC with time [28], which can reach $8^{\circ} \mathrm{C}$ for 1000 hours at $800^{\circ} \mathrm{C}$; (ii) the error due to acquired under high temperatures during long term operation thermoelectric inhomogeneity (henceforth the error due to acquired thermoelectric inhomogeneity), which manifest itself as the dependence of the developed emf on temperature distribution along the TC legs [29]. The latter error is considered to be the most dangerous when measuring temperature using thermocouples: according to [29] it can even reach $30^{\circ} \mathrm{C}$ at the measured temperature of $1000^{\circ} \mathrm{C}$. Recently, there was proposed the TC based sensor to cope with the error due to inhomogeneity [15], [16]. It decreases the error due to inhomogeneity approximately 10 times [15]. However, this sensor does not decrease the error due to drift of TC CC. It just allows using the known methods [27] to decrease this error in a changeable temperature field of a measured object. The error due to drift is quite well studied and even reliable techniques of its prediction exist [27], but the method [27] works only in stable temperature fields and 
does not work in changeable temperature fields. However, the sensor [15], [16] requires methods for temperature control, for instance [30].

Data acquisition systems for TC are being complicated by correction of various errors [24], [25] and implementation of auxiliary procedures [31]-[33]. This makes it difficult to determine their metrological properties. To solve this problem the simulation modeling is widely used [32], [34]. A special software tool, called the metrological software test (MST), was proposed [34]. The MST contains templates of simulation models of typical components of a measuring channel that take into account various components of the error. It allows simulating different values of components of the error, the relations between them, the effect of influence quantities, drifts due to time and temperature, etc. The MST allows determining the reaction of the studied measuring channel to various errors and the efficacy of the error suppression measures. However, the MST presented in [34] is very complex. The goal of this paper is to create the special simplified MST to study the method of determining the TC error in situ during operation [33] (henceforth the method of determining the TC error). The method [33] is possible only for the sensor proposed in [15], [16].

\section{THE METHOD OF DETERMINING THE THERMOCOUPLE ERROR IN SITU DURING OPERATION}

The method of determining the TC error [33] using the purposeful change of a temperature field along the TC legs was proposed. A regular TC (i.e., a TC available on the market and described in [13], [22]), labeled as 1, is shown in Fig.1. It is located in the bore of tubular multi-zone furnace 2. Furnace 2 is attached to the wall of object 3 whose temperature is to be measured. The axis of symmetry is 4 .

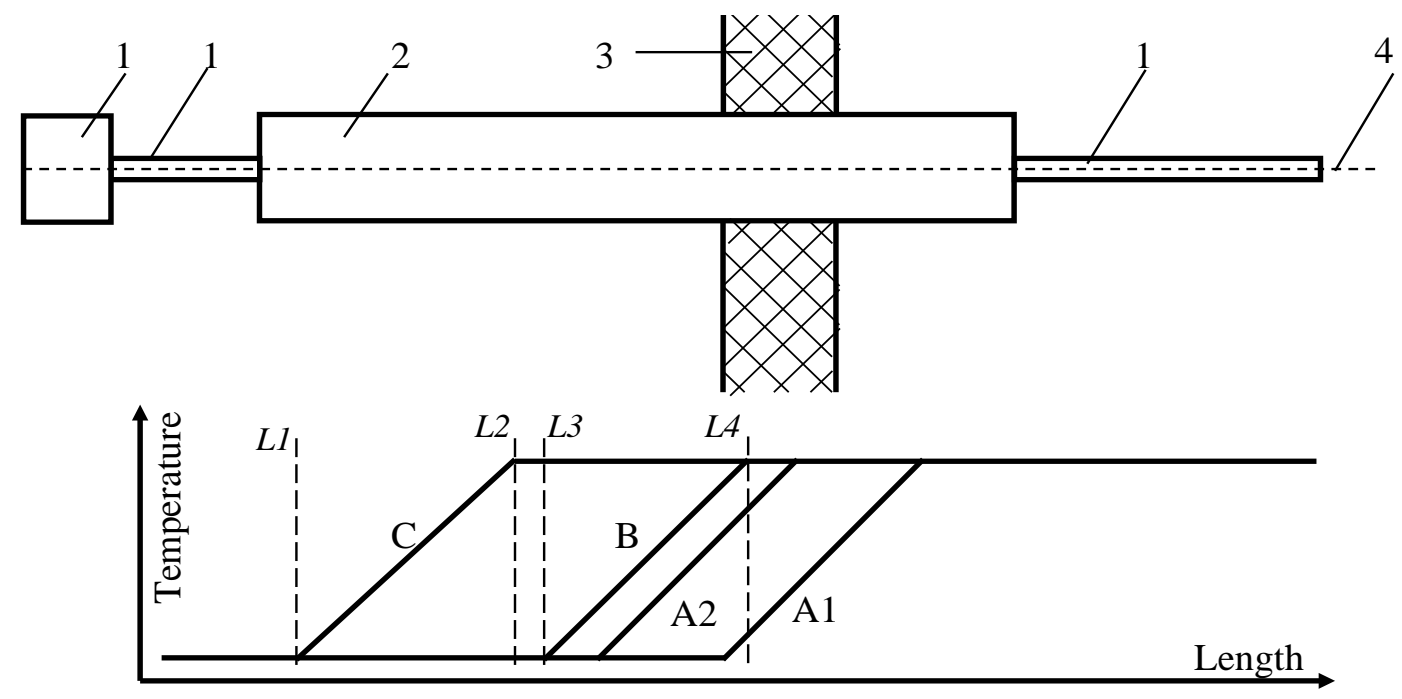

Fig.1. The method of determining the TC error.

The temperature field of object 3 (the temperature field labeled A an the bottom of Fig.1.) changes during operation (for example, within A1 and A2 due to diurnal or weather changes or both simultaneously). In this case, the error due to acquired thermoelectric inhomogeneity appears. Therefore, the first goal of furnace 2 is to stabilize the temperature field profile along the legs of TC 1 . Furnace 2 stabilizes temperature field maintaining the temperature field profile labeled B (see Fig.1., lines L3 and L4). Such stabilization does not make it possible for the error due to acquired thermoelectric inhomogeneity of TC 1 to appear.

Thus, the correction of the TC 1 error is reduced to the correction of the individual deviation of TC $1 \mathrm{CC}$ from the nominal one (based on the calibration before operation) and the correction of the error due to drift of the TC $1 \mathrm{CC}$ with operation time. The methods to correct the error due to drift of a TC CC are known [27]. The method for forecasting the error due to drift of a TC CC using neural networks was proposed [27]. This method allows correcting the individual drift of a TC CC based on the results of determining the TC error. However, the high potential of the method [27] can be only used when a periodic determination of the individual TC error during operation is performed.

Therefore, the second goal of furnace 2 is to carry out a purposeful change in the profile of the temperature field to determine the current error of a TC (the error at a certain instant according to the method described in [33]) in situ during its operation. When determining the TC error, the zone of temperature gradient of the temperature field along TC 1 legs is shifted to the position labeled C (see Fig.1., lines L1 and L2). In the profile of temperature field labeled $\mathrm{C}$, emf is developed by the sections of TC 1 that are usually operated at low temperatures (which means that L3-L2>0). Therefore, the CC of these sections L1-L2 does not undergo degradation and thus does not drift during operation. If the temperature of the TC 1 measuring junction during this procedure remains constant, then the difference between the emfs developed in the profiles $B$ and $C$ equals the error due to drift of the CC of TC 1 [33]. Before the operation of TC 1 , its calibration in the profile of the temperature field $C$ is 
necessary. The emf of TC 1 during this calibration is developed only by the section limited by lines L1-L2. Based on the results of this calibration, one will determine the initial error of the TC. The sum of the initial error and the error due to drift equals the current TC error [33]. The TC 1 error will decrease 8-12 times. Thus, the combination of the methods [27] and [33] ensures the high accuracy of temperature measurements.

\section{THE STRUCTURE OF THE MST FOR STUDYING THE METHOD OF DETERMINING THE THERMOCOUPLE ERROR}

The general structure of the simplified MST, which allows testing the measuring channel in the mode of TC error determination [35], is given in Fig.2. It consists of the following software blocks: (i) the specification of the parameters of profiles of the operation temperature field of the TC and the deviations of the Seebeck coefficient from the nominal one during operation; (ii) the calculation of the temperatures of the endpoints of the virtual sections, into which the $\mathrm{TC}$ is split, during operation $\mathrm{T}_{L i}{ }^{\text {OPER }}$ and during the determination of TC error $\mathrm{T}_{L}^{E R}$ according to the method [33]; (iii) the calculation of the nominal thermo-emf $\mathrm{E}_{i}{ }^{\mathrm{NOM}}$ for the virtual sections; (iv) the calculation of the error of the endpoint temperatures of the virtual sections into which the TC is split caused by the errors of the temperature control systems of multi-zone furnace 2; (v) the calculation of the absolute errors of these virtual sections; (vi) the calculation of the actual thermo-emfs of these virtual sections; (viii) three accumulative adders. The accumulative adders calculate both the net nominal and actual thermo-emfs of the TC and its error based on the calculations of the corresponding parameters of the virtual sections.

To study the influence of separate components on the residual error of TC 1 (the error of the correction of the error due to drift of the CC of TC 1), the coefficients $\mathrm{K}_{C O N T}$ and $\mathrm{K}_{D E G}$ are applied. The coefficient $\mathrm{K}_{C O N T}$ determines the level of the random error in the systems for setting the profile of temperature field of each zone in furnace 2 during operation and calibration. The error $\Delta \mathrm{T}_{C O N T}$ can be randomly simulated by the formula

$$
\Delta T_{\text {CONT }}=K_{\text {CONT }} \cdot(r n d-0.5)
$$

where rnd - uniformly distributed random variable with limits of change from 0 to 1 .

The coefficient $\mathrm{K}_{D E G}$ determines the random deviation of the rate of degradation processes in the virtual sections into which TC 1 is split. The error of each virtual section is randomly simulated by the formula

$$
\Delta T_{D E G}=K_{D E G} \cdot \Delta e_{i} \cdot(r n d-0.5),
$$

where $\Delta e_{i}$ - the deviation of the Seebeck coefficient of the $i$ th section due to degradation of the legs.

The connection block diagram of a temperature measuring system to the MST is given in Fig.3. It includes the MST, whose output generates the nominal value of TC thermoemf $\mathrm{E}_{T P}{ }^{N O M}$, the actual TC thermo-emf (including the error) $\mathrm{E}_{T P}$ and the value of the absolute error of the TC CC $\Delta \mathrm{E}_{T P}$. The values of thermo-emf $\mathrm{E}_{T P}^{N O M}$ and $\mathrm{E}_{T P}$ simulate the thermo-emf developed by the TC in the temperature field of determining the error due to drift of the TC CC and in that of operation. These values are submitted to the temperature measuring system that is currently under test. The random errors of the measuring channel of emf $\Delta_{\Sigma}{ }^{R N D}$ are superimposed with $\mathrm{E}_{T P}{ }^{N O M}$ and $\mathrm{E}_{T P}$ in this system.

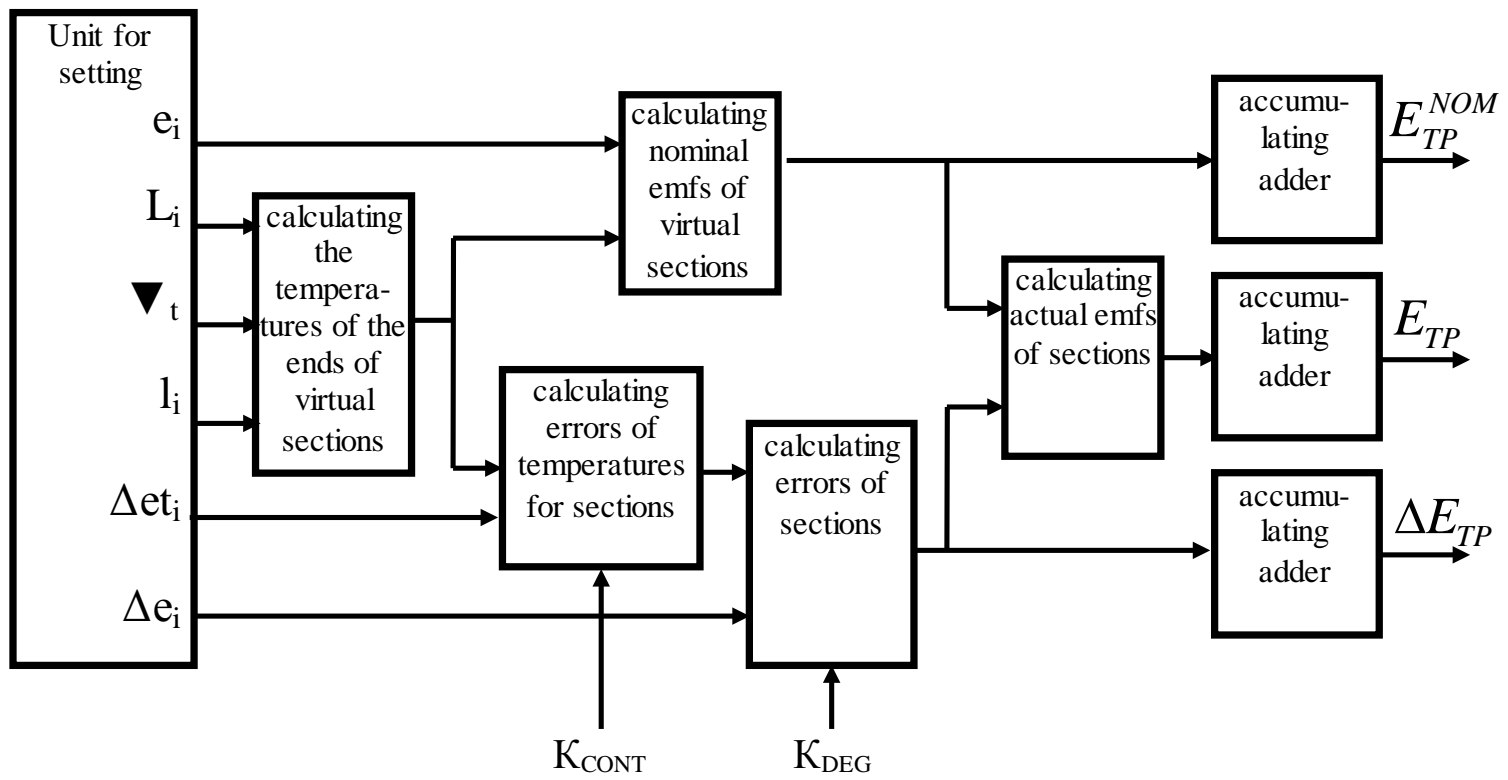

Fig.2. The structure of the simplified MST to test the measuring channel in the mode of determining the TC error during operation. 
The values of the error due to noise $\Delta_{\Sigma}{ }^{\text {NOISE }}$ are randomly simulated similarly to (1). To study the effect of $\Delta_{\Sigma}{ }^{N O I S E}$ on the error determination, the coefficient $\mathrm{K}_{\text {NOISE }}$ similar to the coefficient $\mathrm{K}_{\text {CONT }}$ is applied.

Based on the values of thermo-emfs $\mathrm{E}_{T P}{ }^{N O M}$ and $\mathrm{E}_{T P}$ the temperature measuring system has to determine the error $\Delta \mathrm{E}_{T P}{ }^{S Y S}$ of the main $\mathrm{TC}$, which will be used by the system in the future to correct its error by constructing the individual model of the error due to drift of the TC CC, using, for instance, neural networks as in [27]. The unit analysis of correction error determines the deviation of the error value $\triangle \mathrm{E}_{T P}{ }^{S Y S}$ obtained by the temperature measuring system from the error value $\Delta \mathrm{E}_{T P}$ determined by the MST. The unit analysis of correction error interprets these deviations as the error of determining the error for the main TC.

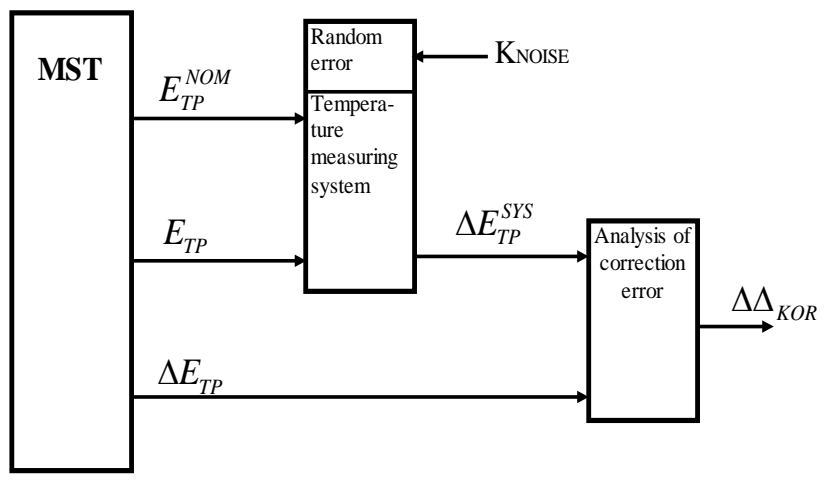

Fig.3. The connection of the temperature measuring system to the MST in the mode of determining the error of the main TC.

\section{THE RESULTS OF THE STUDY}

\section{A. Influence of the error of temperature control}

Firstly, the influence of the error of temperature control of zones in multi-zone furnace 2 (see Fig.1.) on the error of determining the TC error during operation was studied. For this purpose the following values of the coefficient $\mathrm{K}_{C O N T}=0.1 ; 1 ; 10 ; 100$ were considered. These values correspond to the change in the random error of the systems for setting the profile of temperature field for furnace 2 within the interval $\Delta \mathrm{T}_{\mathrm{CONT}} \leq 0.00125 \ldots 1.25^{\circ} \mathrm{C}$ because type $\mathrm{K}$ TCs were studied.

At the same time, the coefficient, which characterizes the rate of degradation in virtual sections, into which TC 1 was split, was given the minimum, that is $\mathrm{K}_{D E G}=0.1$. This means that the rate of degradation in all TC 1 virtual sections was very small, that is, $\Delta \mathrm{T}_{D E G}=0.00125^{\circ} \mathrm{C}$. The coefficient $\mathrm{K}_{\text {NOISE}}$, determining the level of the random error in the measuring channel, was also set as minimum $\mathrm{K}_{N O I S E}=2$. This means that the voltage of the random error of the measuring channel does not exceed $U_{\text {NOISE }} \leq 1 \mu \mathrm{V}$. This corresponds to the temperature error of $\Delta \mathrm{T}_{N O I S E}=0.025^{\circ} \mathrm{C}$.

The results of the study are presented in Fig.4. In this figure, there are four random realizations with the maximum error of determining the TC error for the abovementioned values of $\mathrm{K}_{C O N T}=0.1 ; 1 ; 10 ; 100$ (x-axis), $\mathrm{K}_{D E G}=0.1$ and $\mathrm{K}_{\text {NOISE }}=2$ among 50 realizations.
As can be seen from Fig.4., the errors of the systems for setting the profile of temperature field of furnace 2 virtually do not affect the method of determining the TC error. This statement is in accordance with the theoretical study of the influence of the errors of the systems for setting the profile of temperature field. The errors of these systems affect the error of determining the TC error indirectly. These errors determine only the level of the error due to acquired thermoelectric inhomogeneity. Thus, they are the infinitesimals of higher order with respect to the emf developed by the TC.

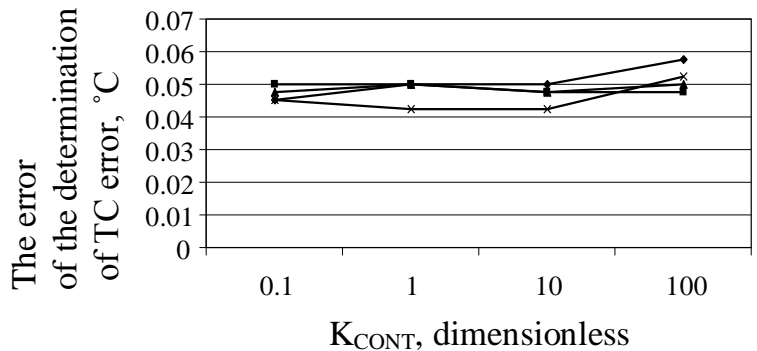

Fig.4. The error of determining the TC error versus the temperature control error of the system of zones in multi-zone furnace 2 .

\section{B. Influence of the rate of degradation}

Then the influence of the rate of degradation in the virtual sections into which TC 1 is split, on the proposed method of determining the TC error was studied. To carry out this study, the coefficient $\mathrm{K}_{D E G}$ was set equal to the following values $K_{D E G}=0.1 ; 1 ; 10$. This corresponds to the change of the Seebeck coefficient of type $\mathrm{K}$ TC within the interval $\Delta \mathrm{T}_{D E G} \leq 0.00125 \ldots 0.225^{\circ} \mathrm{C}$, or on $12.5 \%$. The values of the coefficients $\mathrm{K}_{C O N T}=0.1$ and $\mathrm{K}_{N O I S E}=2$ were set as minimum; therefore, the errors caused by them do not exceed $\Delta \mathrm{T}_{\text {CONT }} \leq 0.00125^{\circ} \mathrm{C}$ and $\Delta \mathrm{T}_{\text {NOISE }} \leq 0.025^{\circ} \mathrm{C}$, respectively.

The results of the study are given in Fig.5. In this figure, there are four realizations with the maximum error of determining the $\mathrm{TC}$ error $\Delta \Delta \mathrm{E}_{T C}$ for the values of the coefficients $\mathrm{K}_{D E G}=0.1 ; 1 ; 10$ (x-axis), $\mathrm{K}_{C O N T}=0.1$ and $\mathrm{K}_{\text {NOISE }}=2$ among 50 realizations.

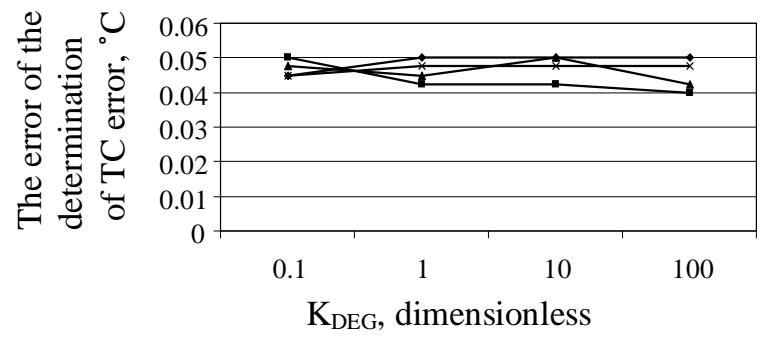

Fig.5. The error of determining the TC error versus the rate of degradation of virtual sections of TC1.

As can be seen from Fig.5., the rate of degradation in the virtual sections, into which TC 1 is split, virtually does not affect the error of determining the TC error during 
operation. This statement is in accordance with the theoretical study. This is because the proposed method of determining the TC error is intended to eliminate the influence of the deviation of the individual rate of degradation in TC legs from the typical one.

\section{Influence of the random error}

The influence of the random error of the measuring channel on the proposed method of determining the TC error was also studied. To carry out this study, the values of the coefficient determining the level of random error were set as follows $K_{N O I S E}=2 ; 10 ; 50$. This corresponds to the change of the random error within the limits $U_{N O I S E}= \pm(1 \ldots 25) \mu \mathrm{V}$, which corresponds to $\Delta \mathrm{T}_{N O I S E}= \pm(0.025 \ldots 0.625)^{\circ} \mathrm{C}$. The values of the coefficients $\mathrm{K}_{C O N T}=0.1$ and $\mathrm{K}_{D E G}=0.1$ were set as minimum; therefore, the errors caused by them do not exceed $\quad \Delta \mathrm{T}_{C O N T} \leq 0.00125^{\circ} \mathrm{C} \quad$ and $\quad \Delta \mathrm{T}_{D E G} \leq 0.00125^{\circ} \mathrm{C}$, respectively.

The results of the study are given in Fig.6. In this figure there are realizations with the maximum and minimum errors of the error of determining the TC error $\Delta \Delta \mathrm{E}_{T C}$ for the values of the coefficients $\mathrm{K}_{N O I S E}=2 ; 10 ; 50$ (x-axis), $\mathrm{K}_{C O N T}=0.1$ and $\mathrm{K}_{D E G}=0.1$ among 50 realizations. As can be seen from Fig.6., the error of determining the TC error during operation rises strongly with the rise of the random error of a measuring channel. This rise is virtually proportional to the rise of the random error. Thus, the main source of the error of measurements of the TC CC during the procedure of determining the TC error during operation is the random error of the measuring channel of emf.

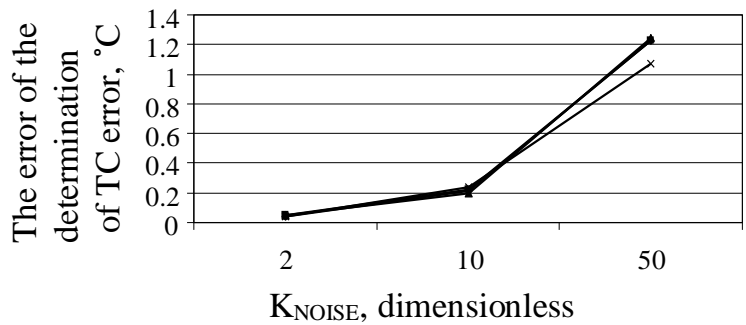

Fig.6. The error of determining the TC error versus the random error of the measuring channel.

\section{Decreasing the influence of common and normal mode noises}

It should be noted that the random error of a measuring channel of emf usually consists of two components: (i) the noise of the input amplifier of TC emf; (ii) the residual error due to the normal and common mode noises. The effect of the input amplifier's noise can be reduced by an integrated analog-to-digital converter (in particular, dual slope integration or sigma-delta analog to digital converters) and a high-quality operational amplifier.

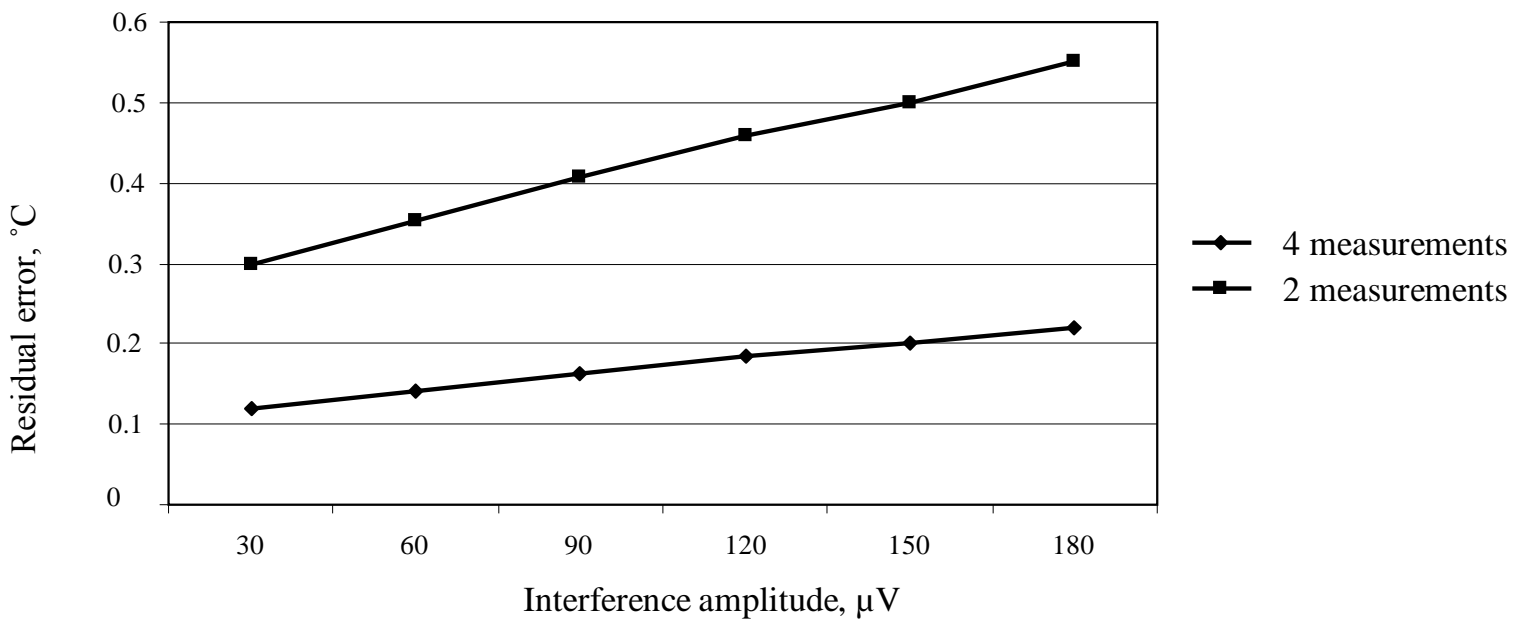

Fig.7. The error of determining the TC error for the discrete averaging of two and four measurement results versus interference amplitude.

Further reduction of the random error can be performed by averaging the results of several replicate measurements. Due to the fact that the input amplifier's noise is close to the white noise, the coefficient of reduction of its effect is proportional to $\mathrm{n}^{0.5}$, where $\mathrm{n}$ is the number of averaged replicate measurements [36]. The residual error due to the normal and common mode noises is caused by the power grid delivering power supply to a measuring channel. Therefore, within a series of sequential measurements they have virtually deterministic nature. This fact makes it possible to improve the efficiency of averaging the results of several replicate measurements by increasing the time of measurements. A simpler method for improving the accuracy is averaging of an even number of replicate measurements [37]. In this case, the beginning of each measurement has to be synchronized with the corresponding phase of the power grid voltage. For instance, it is reasonable to average the results of two measurements 
whose beginnings are shifted in time on an odd number of halves of the power grid period. Thus, the residual interference during each measurement is shifted by $180^{\circ}$. The error of determining the TC error in this case is given in Fig.7. (the upper straight line) among 50 realizations. The error in this case, in comparison with the errors given in Fig.6., is approximately two times lower, whereas the amplitude of the interference is about four times greater. Even greater accuracy can be achieved by averaging four measurement results, whose beginnings are shifted on an odd number of quarters of the power grid period. Then, the residual interference during each measurement is shifted by $90^{\circ}$. The error of determining the TC error in this case is also given in Fig.7. (the bottom line). As can be seen from Fig.7., the error of determining the TC error decreases even more than twice.

\section{CONCLUSIONS}

As it is shown in the paper, the random error as well as the normal and common mode noise has considerable effect on the proposed method of determining the TC error [33]. They can significantly increase the error of determining the TC error using the proposed method. The noise and interference of the order of $5-6 \mu \mathrm{V}$ is still acceptable. They cause the error of about $0.2-0.3^{\circ} \mathrm{C}$. Therefore, high noise immunity is a necessary condition for high accuracy of TC error correction and, consequentially, for accurate temperature measurements.

It should be noted that this paper considers random components of the error of determining the TC error in situ during operation proposed in [33]. The influence of systematic components is considered in [33]. Based on this study as well as on the study [33] the uncertainty budget can be composed for the method of determining the TC error.

As shown in this paper, the effect of the rate of degradation processes and the error of control of temperature field is of the order of few hundredths of a Celsius degree. This effect can be neglected.

It is known that the errors of sensors, in particular TCs, dominate in a measuring channel. A system that uses the investigated method can considerably (ten times or even more) reduce the intercalibration interval. In this case, calibrations occur during the operation. Thus, the accuracy of the temperature measurement and the metrological reliability of the system can be considerably improved. Due to the fact that the investigated method does not require the use of additional equipment (in particular, standards), it significantly increases the metrological autonomy of such systems.

\section{ACKNOWLEDGMENT}

This work was supported by Green Industry Technology Leading Project of Hubei University of Technology (No. ZZTS2016004); Foundation of Wuhan Science and technology Bureau (2015030809020370); Doctoral Scientific Research Fund from Hubei University of Technology (No. BSQD14037).

\section{REFERENCES}

[1] Józwik, J., Mika, D. (2015). Diagnostics of workpiece surface condition based on cutting tool vibrations during machining. Advances in Science and Technology Research Journal, 9 (26), 57-65.

[2] Glowacz, A., Glowacz, Z. (2016). Diagnostics of stator faults of the single-phase induction motor using thermal images, MoASoS and selected classifiers. Measurement, 93, 86-93.

[3] Fraczyk, A., Kucharski, J. (2017). Surface temperature control of a rotating cylinder heated by moving inductors. Applied Thermal Engineering, 125, 767779.

[4] Krolczyk, G.M., Maruda, R.W., Nieslony, P., Wieczorowski, M. (2016). Surface morphology analysis of Duplex Stainless Steel (DSS) in Clean Production using the Power Spectral Density. Measurement, 94, 464-470.

[5] Kočí, V., Maděra, J., Jerman, M., Trník, A., Černý, R. (2014). Determination of the equivalent thermal conductivity of complex material systems with largescale heterogeneities. International Journal of Thermal Sciences, 86, 365-373.

[6] Novák, M., Náprstková, N., Józwik, J. (2015). Analysis of the surface profile and its material share during the grinding inconel 718 alloy. Advances in Science and Technology Research Journal, 9 (26), 4148.

[7] Wang, W.L., Zhang, H., Du, X.J., Sun, Y.Y. (2016). PCB-integrated thin film thermocouples for transient temperature measurement. Electronics Letters, 52 (13), 1140-1141.

[8] Mikhalieva, M., Hots, N., Mykyychuk, M., Dzikovska, Y. (2017). Use electric and acoustic technologies for automated control of water. In Advances in Intelligent Systems and Computing: Selected Papers from the International Conference on Computer Science and Information Technologies. Springer, Vol. 512, 293303.

[9] Feldshtein, E., Józwik, J., Legutko, J. (2016). The influence of the conditions of emulsion mist formation on the surface roughness of AISI 1045 steel after finish turning. Advances in Science and Technology Research Journal, 10 (30), 144-149.

[10] Mellal, I., Laghrouche, M., Tien Bui, H. (2017). Field programmable gate array (FPGA) respiratory monitoring system using a flow microsensor and an accelerometer. Measurement Science Review, 17 (2), 61-67.

[11] Ferrero, A., Scotti, V. (2013). Forensic metrology: A new application field for measurement experts across techniques and ethics. IEEE Instrumentation \& Measurement Magazine, 16 (1), 14-17.

[12] Birch, J. (2003). Benefit of legal metrology for the economy and society. A study for the International Committee of Legal Metrology. https://www.oiml.org/en/files/pdf_e/e002-e03.pdf.

[13] Webster, J. (1999). Measurement, Instrumentation, and Sensors Handbook. CRC Press. 
[14] Childs, P.R.N., Greenwood, J.R., Long, C.A. (2000). Review of temperature measurement. Review of Scientific Instruments, 71, 2959-2978.

[15] Kochan, O., Kochan, R., Bojko, O., Chyrka, M. (2007). Temperature measurement system based on thermocouple with controlled temperature field. In 4th IEEE Workshop on Intelligent Data Acquisition and Advanced Computing Systems: Technology and Applications (IDAACS 2007), 6-8 September 2007. IEEE, 47-51.

[16] Jun, S., Kochan, O., Kochan, V., Wang, C. (2016). Development and investigation of the method for compensating thermoelectric inhomogeneity error. International Journal of Thermophysics, 37 (1).

[17] Maruda, R.W., Krolczyk, G.M., Nieslony, P., Wojciechowski, S., Michalski, M., Legutko, S. (2016). The influence of the cooling conditions on the cutting tool wear and the chip formation mechanism. Journal of Manufacturing Processes, 24 (1), 107-115.

[18] Glowacz, A., Glowacz, A., Glowacz, Z. (2015). Recognition of thermal images of direct current motor with application of area perimeter vector and bayes classifier. Measurement Science Review, 15 (3), 119126.

[19] Stadnyk, B., Yatsyshyn, S., Skoropad, P. (2012). Research in nanothermometry. Part 4. Amorphous alloys of thermo-resistive thermometry. Sensors and Transducers, 141 (6), 1-7.

[20] Machin, G., Bojkovski, J., del Campo, D. et al. (2014). A European roadmap for thermometry. International Journal of Thermophysics, 35 (3-4), 385-394.

[21] Palenčár, R., Sopkuliak, P., Palenčár, J., Ďuriš, S., Suroviak, E., Halaj, M. (2017). Application of Monte Carlo Method for evaluation of uncertainties of ITS-90 by Standard Platinum Resistance Thermometer. Measurement Science Review, 17 (3), 108-116.

[22] Park, R.M. (1993). Manual on the Use of Thermocouples in Temperature Measurement. ASTM International.

[23] Smalcerz, A., Przylucki, R. (2013). Impact of electromagnetic field upon temperature measurement of induction heated charges. International Journal of Thermophysics, 34 (4), 667-679.

[24] Kochan, R., Kochan, O., Chyrka, M., Vasylkiv, N. Precision data acquisition (DAQ) module with remote reprogramming. In IEEE Intelligent Data Acquisition and Advanced Computing Systems: Technology and Applications (IDAACS 2005), 5-7 September, 2005. IEEE, 279-282.

[25] Duan, Q. (2009). Research of a cold end temperature compensation for thermal couple. In IEEE International Conference on Automation and Logistics (ICAL '09), 5-7 August, 2009. IEEE, 921-924.
[26] Körtvélyessy, L. (1998). Thermoelement Praxis. Essen, Germany: Vulkan-Verlag. (in German).

[27] Sachenko, A., Kochan, V., Turchenko, V. (2000). Sensor drift prediction using neural networks. In International Workshop on Virtual and Intelligent Measurement Systems (VIMS 2000), 29-30 April, 2000, 88-92.

[28] Rogelberg, N., Nuzhnov, A., Pokrovskaya, G. et al. (1969). The stability of the chromel-alumel thermocouples' thermoelectric power at temperatures up to $1200^{\circ} \mathrm{C}$. Investigation of alloys for thermocouples. In Proceedings of the Giprotsvetmetobrabotka.

[29] Sloneker, K.C. (2009). Thermocouple inhomogeneity. Ceramic Industry, 159 (4), 13-18.

[30] Vasylkiv, N., Kochan, O., Kochan, R., Chyrka, M. (2009). The control system of the profile of temperature field. In IEEE International Workshop on Intelligent Data Acquisition and Advanced Computing Systems (IDAACS 2009), 21-23 September, 2009. IEEE, 201-206.

[31] Jun, S., Kochan, O., Kochan, R. (2016). Thermocouples with built-in self-testing. International Journal of Thermophysics, 37 (4).

[32] Woolley, J.W., Woodbury, K.A. (2009). Using computational models to account for thermocouple conduction error in cast metal/mold interfacial heat transfer experiments. In 113th Metalcasting Congress, 7-10 April 2009, Vol. 117, 31-40.

[33] Shu, C., Kochan, O. (2013). Method of thermocouples self verification on operation place. Sensors \& Transducers, 160 (12), 55-61.

[34] Vasylkiv, N. (2010). Improvement of metrology software test in computer systems of temperature measurement. International Journal of Computing, 9 (2), 175-182.

[35] Jun, S., Kochan, O., Levkiv, M. (2017). Metrological software test for studying the method of thermocouple error determination during operation. In 11th International Conference on Measurement (Measurement 2017), 29-31 May, 2017. Bratislava, Slovakia: Institute of Measurement Science SAS, 171174.

[36] International Organization for Standardization. (2010). Guide to the Expression of Uncertainty in Measurement (GUM).

[37] Informa USA, Inc. Electronic Design. http://www.electronicdesign.com/analog/when-1-1-3db-averaging-adc-channels-improve-nsd.

Received September 22, 2017. Accepted March 20, 2018. 